The following paper posted here is not the official IEEE published version. The final published version of this paper can be found in the Proceedings of the International Geoscience and Remote Sensing Symposium (2005 : Seoul, Korea): pp.1097-1100

Copyright (C) 2005 IEEE.

Personal use of this material is permitted. However, permission to reprint/republish this material for advertising or promotional purposes or for creating new collective works for resale or redistribution to servers or lists, or to reuse any copyrighted component of this work in other works must be obtained from the IEEE. 


\title{
The Development of a Ground Based Polarimetric SAR Interferometer (GB-POLInSAR)
}

\author{
Zheng-Shu Zhou, Shane R Cloude \\ School of Electrical and Electronic Engineering \\ University of Adelaide \\ SA 5005, Australia \\ zszhou@eleceng.adelaide.edu.au
}

\begin{abstract}
In this paper we present our ideas for the development of a new type of ground based vector radar interferometer system. This concept differs from other sensors by virtue of combining coherent imaging polarimetry with interferometry. We first describe the system concept and then present an analysis of results to illustrate the potential new applications of such a sensor.
\end{abstract}

\section{INTRODUCTION}

Polarimetric Interferometric Synthetic Aperture Radar (POLInSAR) has been developed mainly for applications in space and airborne SAR systems [1] and little attention has been paid to its potential role in ground-based observations [2]. Nonetheless, it has several potential advantages over existing ground based sensing methods and in this paper we outline the results of an initial study into the requirements for deployment of such a ground based vector interferometer. Ground based radar observations are not new. Various scatterometers [3], imaging GB-SAR systems [4, 5] and 3-D imagers [2, 6] have been developed and deployed. More recently ground-based interferometers have also been widely used for monitoring subsidence and other small displacements [7]. The key added benefits of a GB-POLINSAR system over these existing sensors are:

a) As an instrument for robust quantitative studies of vegetation cover. GB-POLInSAR systems provide for easier deployment of broad-band and multi-baseline techniques, from which we can estimate vegetation structure and extinction propagation using model based techniques. There is also the potential to study sub-canopy surface parameters such as soil moisture [8]. These are important, both for developing new algorithms for application to future air and space borne sensors, but also in their own right for the deployment of local sensors in monitoring vegetation growth etc. This micro-scale multiparameter combination with good temporal resolution is a unique feature of ground-based sensors.

b) GB-POLInSAR systems provide the potential for extracting 3-D structural information much faster than full 3-D scans, which is important for vegetation applications in the field, where temporal motion effects can defocus 3-D images. One disadvantage of POLInSAR is the requirement for robust model-based parameter estimation algorithms using baseline and frequency coherence variation. Therefore, in this paper we investigate both the system requirements for such a sensor as well as presenting an initial study into the robustness of existing coherence based parameter retrieval models for application in a GB environment.

\section{POLINSAR SYSTEM DESIGN ISSUES}

To address the above problems, a GB-POLInSAR system is under development at the University of Adelaide.

\section{A. Principle of GB-SAR and Resolution}

In a ground-based arrangement, a synthetic aperture can be obtained by scanning the antenna on a linear horizontal mechanical guide and/or a vertical guide. In our case the scan along track provides image azimuth spatial resolution; the vertical displacement contributes to interferometric baseline. The range resolution is provided by the broadband SAR synthesis algorithm of radar signals and depends on the radar bandwidth. As the radar image is obtained through synthesis and sampling techniques, its characteristics are constrained by the radar measuring parameters: bandwidth, frequency step, scan aperture length, and scan interval. Therefore, the azimuth resolution $D_{a}$ and the range resolution $D_{r}$ are given by the following equations [9].

$$
\begin{aligned}
& D_{a}=\frac{c}{2 L_{a} f} \sqrt{\left(\frac{L_{a}}{2}\right)^{2}+R^{2}} \\
& D_{r}=\frac{c}{2 B} \csc \theta
\end{aligned}
$$

where

$c$ - the speed of light

$L_{a}$ - the total horizontal scan aperture of the antenna

$\theta$ - the incidence angle

$R$ - the range distance

$f$ - the radar center frequency

$B$ - the radar bandwidth.

This work was partially supported by the DSTO, Australia. 
For the broadband radar system case, particularly for us in C-band from $4 \mathrm{GHz}$ to $6 \mathrm{GHz}, 2 \mathrm{GHz}$ of bandwidth can be used for achieving range resolution of $7.5 \mathrm{~cm}$, which is generally satisfactory in practical application. The corresponding azimuth resolution will be $11 \mathrm{~cm}$ with azimuth aperture of $10 \mathrm{~m}$ and range distance of $20 \mathrm{~m}$.

\section{B. System Schematic and Procedures}

Fig. 1 shows the schematic configuration of a proposed GB-POIInSAR system. A Performance Network Analyzer (PNA) based system provides extra-wide frequency range, multi-bands and repeatable data acquisition configurations. We will use a vehicle to deploy all equipment and move along track to realize the synthetic aperture. Sensors are lifted up and look-down along the cross-track direction. By using a dual polarized diagonal horn antenna, as shown in Fig. 2, the full set of coherent polarization components $\mathrm{HH}, \mathrm{HV}, \mathrm{VH}$, and $\mathrm{VV}$ data can be acquired 'quasi' simultaneously using the up and down sweeps of the PNA. To enable interferometric measurement, we propose two possible methods.

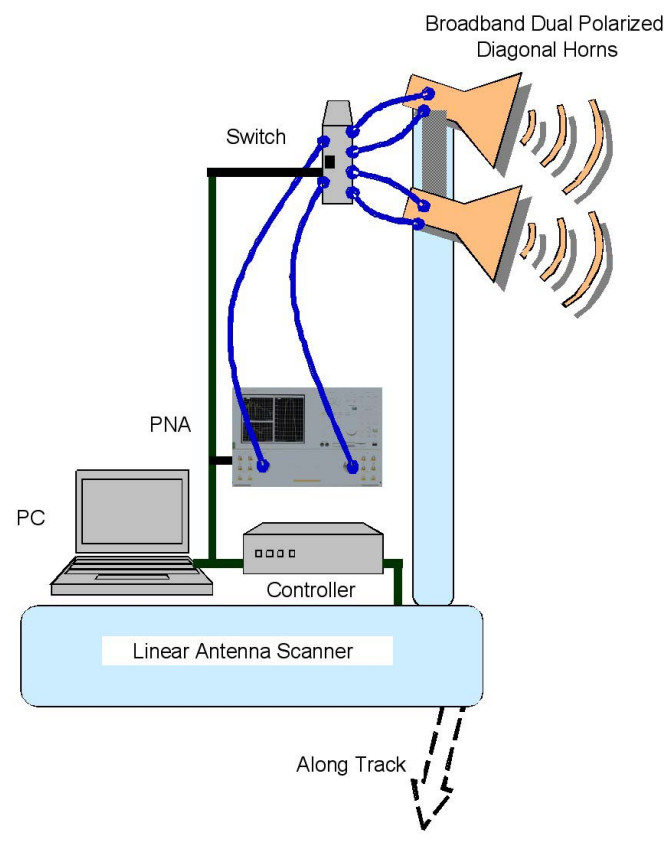

Figure 1. System Schematic of GB-POLInSAR

One method is to set up two identical antennas separated by the required baseline and an electronic switch. At each azimuth position, switch to sensor 1 and acquire one polarization dataset, then switch to sensor 2 and acquire another dataset. This approach is also scalable to multiple antennas in an array for switching multiple baselines.

Another approach is to use one antenna only. At the same azimuth position, we change elevation of the sensor to a different position (the difference being the baseline of the interferometric SAR) and acquire quad polarization datasets at each. This provides a cheaper more compact system design but requires longer time window for data capture.

We have carried out some calibration tests in our anechoic chamber (Fig. 2). A dihedral corner reflector in multiple orientations was employed as a polarimetric calibrator for the system. The system demonstrates good stability and repeatability with xpol isolation better than $-25 \mathrm{~dB}$ and phase and amplitude imbalances less than 5 degree and $0.2 \mathrm{~dB}$ across the band, respectively.

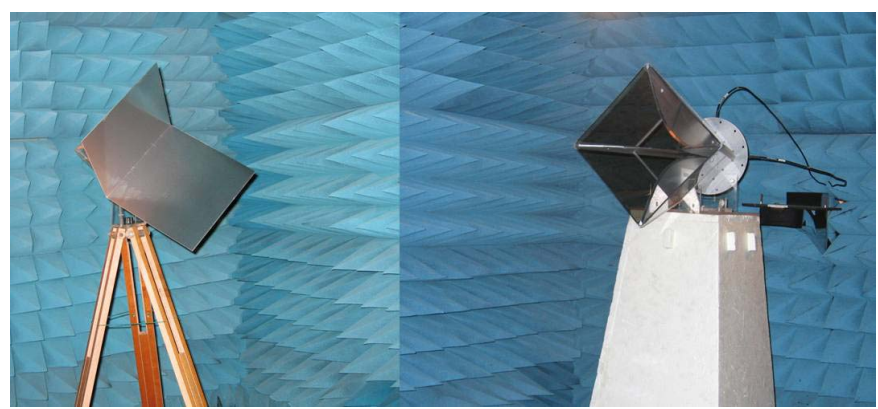

Figure 2. Dual polarized Diagonal Horn Antenna and Dihedral Calibrator in the Anechoic Chamber

The developed system will be deployed for monitoring and detecting different vegetation features. Therefore, the following design objectives should be met:

a) System development and evaluation with high accuracy of polarimetric calibration;

b) Realization of POIInSAR functions with ideal targets in the anechoic chamber;

c) Application for vegetation height estimation in the field with satisfactory resolution and accuracy;

d) Potential ground truth validation for airborne or spaceborne SAR.

To motivate the development of such a system we now turn to consider the robustness of retrieval models for GBPOLInSAR.

\section{RETRIEVAL MODELS FOR GB-POLINSAR}

Current POLInSAR applications are centred on vegetation parameter estimation based on the variation of interferometric coherence with polarization. As an example, the orientedvolume-over-ground (OVOG) two-layer model describes the coherence of vegetated terrain for a triplet of polarisation channels (usually $\mathrm{VV}, \mathrm{HV}$ and $\mathrm{HH}$ ) in terms of a set of important vegetation structure parameters as shown in (2) [10]

$$
\tilde{\gamma}_{p q}=e^{i \phi} \frac{\gamma_{v}\left(\sigma_{p}+\sigma_{q}, h_{v}\right)+\mu_{p q}}{1+\mu_{p q}}
$$

Here there are six (three complex) observations, namely the phase and coherence of $\mathrm{HH}, \mathrm{HV}$ and $\mathrm{VV}$ interferograms and these are related to 7 physical parameters of interest, namely

$$
\begin{aligned}
& h_{v}-\text { the vegetation height } \\
& \phi-\text { the ground topographic phase } \\
& \sigma_{h}-\text { extinction for horizontal polarised waves }
\end{aligned}
$$


$\sigma_{v}-$ the extinction for vertical polarised waves

$\mu_{\mathrm{hh}}, \mu_{\mathrm{hv}}$ and $\mu_{\mathrm{vh}}-$ the surface-to-volume scattering ratios

$\sigma$ and $\mu$ in particular are functions of frequency that contain important micro-scale information on vegetation and surface properties.

\section{ANALYSIS OF GB-POLINSAR DATA}

The data set selected for OVOG model investigation is an indoor measurement (hence free of temporal decorrelation) of a maize sample collected at the JRC-EMSL facility [6]. The sample consists of a tall stand of $6 \times 6$ plants with a height of $1.8 \mathrm{~m}$ planted in a square container of size $2 \mathrm{~m}$. Fig. 3 shows an image of the test sample.

The measurement geometry inside the EMSL anechoic chamber is shown in Fig. 4. The vegetation sample is placed on a rotating turntable so that measurements can be made over 360 degrees of azimuth for a given angle of incidence. The antenna beamwidth is such that the sample is uniformly illuminated by the transmitter. In this experiment there were 72 azimuth steps of 5 degrees. At each position the frequency is stepped across the frequency range 1.5 to $9.5 \mathrm{GHz}$ (in $10 \mathrm{MHz}$ steps) and the elevation angle incremented in 0.25 degree steps from 44 to 45 degrees. In this way interferometric analysis can be performed with a minimum baseline of 0.25 degrees. Note finally that the focus for the chamber (zero phase position for interferometry) is located around $38 \mathrm{~cm}$ above the ground surface of the sample. Hence the ground phase parameter $\phi$ in (2) will not be zero and will change with frequency and baseline.

The processing of the wide band signals starts with the two calibrated complex signals $s_{1,2}$ from positions $\theta$ and $\theta+\Delta \theta$ as shown in (3)

$$
s_{1}=E_{\theta}^{p q}(f) \quad s_{2}=E_{\theta+\Delta \theta}^{p q}(f)
$$

The wide band interferogram is then formed from the product of common spectral band filtered and phase offset signals as shown in (4).

$$
s_{1} \cdot s_{2}^{*}=E_{\theta}^{p q}(f) \cdot \operatorname{conj}\left(E_{\theta+\Delta \theta}^{p q} f-\frac{f \Delta \theta}{\tan \theta}\right) e^{-i \frac{4 \pi f \Delta \theta}{c \sin \theta} z_{o}}
$$

Finally the complex coherence for polarization combination $p q$ and frequency $f$ is calculated as

$$
\tilde{\gamma}_{p q}(f)=\frac{\left\langle s_{1} s_{2}^{*}\right\rangle}{\sqrt{\left\langle s_{1} s_{1}^{*}\right\rangle\left\langle s_{2} s_{2}^{*}\right\rangle}}
$$

In SAR modes, the averaging is made over neighbouring pixels but in this non-imaging case averaging is made over 360 degrees of azimuth coverage combined with frequency smoothing over a $320 \mathrm{MHz}$ bandwidth. Fig. 5 shows the resulting variation of complex coherence in (5) with frequency. Note the following points:

a) The ground phase has been normalized to zero for all frequencies by (4).

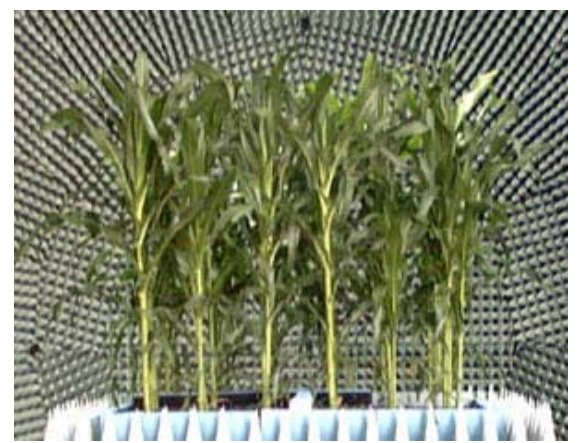

Figure 3. Image of Test Maize Sample in JRC-EMSL Facility

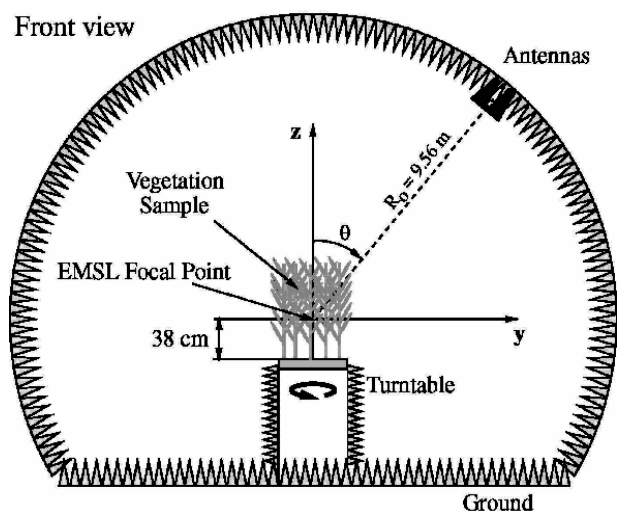

Figure 4. Schematic of POIInSAR Measurements at the JRC-EMSL Facility

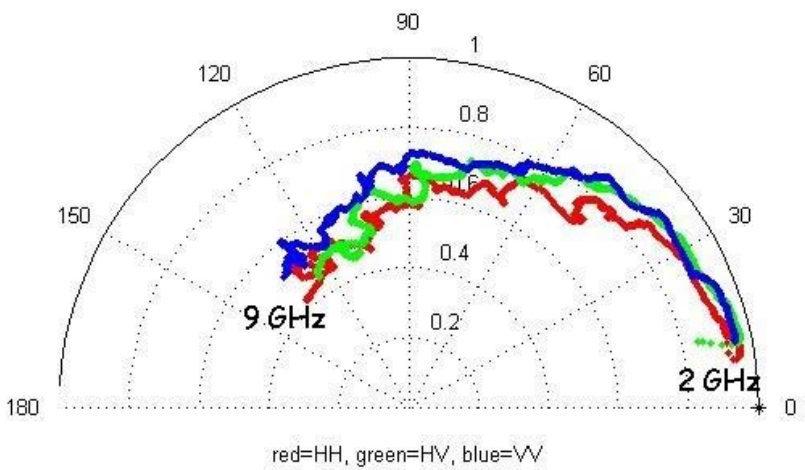

Figure 5. Variation of Interferometric Coherence with Frequency for the JRC EMSL Maize Sample

b) The vegetation bias increases with frequency at the same time as the coherence magnitude reduces. This is characteristic of the presence of volume decorrelation, the physical mechanism supporting the OVOG model.

We note also that the coherence is polarization dependent. However, this dependence can be due to two very different physical processes, namely differential extinction $\sigma$ or polarization variation of surface scattering through $\mu$ in (2). To investigate further, we show in Fig. 6 the variation of coherence amplitude with frequency. Also shown as the dashed line is the zero extinction 'sinc' model for coherence (a degenerate example of the OVOG model). This represents an 
important comparison of the data, as the coherence can be lower than this curve only through the presence of surface scattering contributions, while it lies above this line in the presence of strong wave extinction effects. We see that up to around $6 \mathrm{GHz}$ the coherence values lie below the 'sinc' curve, indicating surface component effects. Interpretation above 6 $\mathrm{GHz}$ is made difficult because of problems of coherence bias at low coherence values i.e. the coherence can be overestimated at low coherence due to insufficient number of looks.

This conclusion is reinforced by reference to Fig. 7. Here we show the height of the phase centre in the vegetation as a function of frequency. Note that the 'sinc' model predicts a phase centre at half the vegetation height $(0.9 \mathrm{~m}$ in this case). The phase centre can only be lower than this level because of the presence of surface scattering contributions $(\mu)$ pulling the phase centre closer to the ground. When the phase centre is above the 'sinc' level, then extinction $(\sigma)$ becomes dominant over surface scattering effects. Significantly we see that below $5 \mathrm{GHz}$ the surface effects dominate over extinction with $\mathrm{HH}$ having a larger surface contribution than VV. Above $5 \mathrm{GHz}$ the phase centre lies close to the 'sinc' line and again detailed interpretation of the phase variations is made difficult by the low coherence at higher frequencies. Development of our own GB system will enable us to investigate these issues in more detail and to develop new retrieval algorithms for extinction, structure and surface parameter estimation.

\section{CONCLUSIONS}

In this paper a new GB-POLInSAR system is introduced and a study made to demonstrate the robustness of coherence based parameter retrieval models for application in a GB environment. PNA-based GB-POLInSAR systems provide for easier deployment of broad-band and multi-baseline techniques, from which we propose to estimate vegetation structure, extinction and surface parameter retrieval using model based techniques. This micro-scale multi-parameter combination with good temporal resolution is a unique feature of ground-based sensors. Our ongoing work will demonstrate several new applications of GB-POLInSAR, aimed at extracting 3D structural information much faster than full $3 \mathrm{D}$ scans and developing new algorithms for application to future air and space borne sensors.

\section{ACKNOWLEDGEMENT}

The authors wish to acknowledge the kind support of the JRC Ispra for use of their EMSL chamber data in this project.

\section{REFERENCES}

[1] K. P. Papathanassiou, S. R. Cloude, "Single Baseline Polarimetric SAR Interferometry," IEEE Trans. Geosci. Remote Sensing, vol. 39, pp. 2352-2363, November 2001.

[2] Z.-S. Zhou, W. M. Boerner, M. Sato "Development of a Broadband Ground Based Polarimetric SAR System for Noninvasive Ground-Truth Validation in Vegetation Monitoring," IEEE Trans. Geosci. Remote Sensing, vol. 42, pp. 1803-1810, September 2004.

[3] B. Brisco, R. J. Brown, J. G. Gairns and B. Snider, "Temporal GroundBased Scatterometer Observations of Crops in Western Canada," Canadian Journal of Remote Sensing, vol. 18, pp. 14-21, January 1992.
[4] M. Pieraccini, G. Luzi, C. Atzeni, "Terrain Mapping by Ground-Based Interferometric Radar," IEEE Trans. Geosci. Remote Sensing, vol. 39, pp. 2176-2181, October 2001.

[5] K. Morrison, J. C. Bennett, G. Cookmartin, S. Quegan, and A. Race, "Developemnt and Capabilities of the Ground-Based SAR (GB-SAR) Facility," in Proceedings of the Advanced SAR Workshop, Canadian Space Agency, Montreal, 25-26 June, 2003, CD-ROM

[6] L Sagues, J M Lopez, J Fortuny, X Fabregas, A Broquetas, A J Sieber, "Indoor Experiments on Polarimetric SAR Interferometry," IEEE Trans. Geosci. Remote Sensing, vol. 38, pp. 670-684, March 2000.

[7] D. Leva, G. Nico, D. Tarchi, J. Fortuny-Guasch and A. J. Sieber "Temporal Analysis of a Landslide by Means of a Ground-Based SAR Interferometer," IEEE Trans. Geosci. Remote Sensing, vol. 41, pp. 745752, April 2003.

[8] S. R. Cloude , M. L. Williams, "Estimating sub-canopy soil moisture using POLInSAR," in Proceedings of $2^{\text {nd }}$ ESA POLInSAR Workshop, Frascati, January 2005, http://earth.esa.int/workshops/polinsar2005/

[9] H. Rudolf, D. Tarchi, and A. J. Sieber, "Combination of Linear and Circular SAR for 3-D Features," in Proc of IGARSS 1997, Singapore 3-8 August 1997, pp. 1551-1553.

[10] R. N. Treuhaft, S. R. Cloude, "The Structure of Oriented Vegetation from Polarimetric Interferometry", IEEE Trans. Geosci. Remote Sensing, vol. 37, pp. 2620-2624, September 1999.

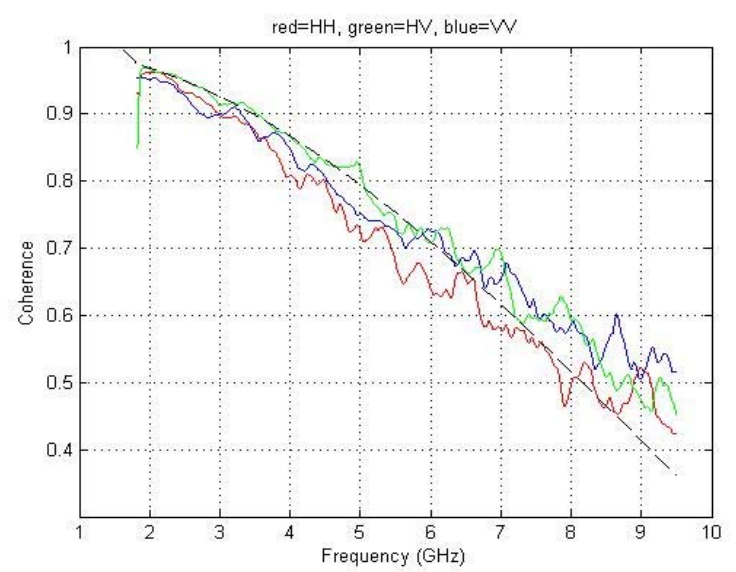

Figure 6. Coherence Amplitude Variation vs. Frequency (zero extinction case shown as dashed line)

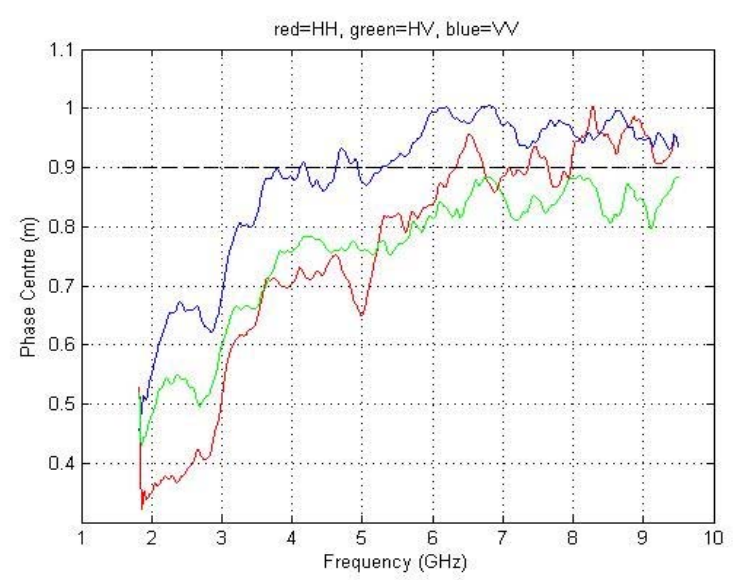

Figure 7. Phase Centre Variation as a Function of Frequency 